\title{
DISCRETE SUBGROUPS OF THE LORENTZ GROUP
}

\author{
LEON GREENBERG
}

\section{Introduction.}

Let $D$ be the unit disk $\{z|| z \mid<1\}$ of the plane of complex numbers, $E$ its boundary and $\mathfrak{Q}^{+}$the group of linear fractional transformations which leave $D$ invariant. The following has been proved by J. Nielsen [3].

TheOREM A. If $\mathbb{B S}$ is a non-commutative subgroup of $\mathfrak{L}^{+}$which contains only hyperbolic transformations (besides the identity), then (B) is discrete.

This theorem has been generalized in several ways. W. Fenchel and J. Nielsen [2] and C. L. Siegel [4] have proved the following. (The Fenchel-Nielsen formulation is slightly different.)

Theorem B. If (S) is a subgroup of $\mathfrak{L}^{+}$such that

a) the elements of \&S do not all leave invariant a point or pair of points in $E$,

b) the identity is not a limit point of the elliptic elements of $\mathbb{S S}$, then $\mathbb{S S}$ is discrete.

W. T. Van Est [1] has proved the following.

Theorem C. Let $\mathfrak{F}$ be a semi-simple Lie group with trivial center and with Lie algebra $\mathscr{F}$. Let SS be a subgroup of $\mathfrak{F}$ which satisfies the following conditions.

a) There is a neighborhood $U$ of 0 (in $\mathscr{F}$ ) so that if $X \in U, X \neq 0$ and $\exp X \in \mathbb{B S}$, then the Killing form $\langle X, X\rangle>0$.

b) If $X \in \mathscr{F}$ and $\exp X \in \mathbb{S}$, then $\operatorname{ad} X$ is not nilpotent.

Then the closure $\overline{\mathbb{S}}$ contains a normal subgroup $\mathfrak{N}$ of finite index, such that $\mathfrak{R}=\mathfrak{C} \times \mathfrak{D}$, where $\mathfrak{C}$ is an open, connected, central, Lie subgroup of $\mathfrak{N}$, and D is discrete.

The purpose of this paper is to investigate in greater detail the case where $\mathfrak{F}$ is the group of rigid motions of $n$-dimensional hyperbolic space. (We shall denote this group by $\mathfrak{L}$ in the following. $\mathfrak{L}$ is isomorphic to a subgroup of index 2 in the Lorentz group in $n+1$ variables.) Several

Received September 12, 1960.

This work has been supported by the United States Office of Naval Research. 
analogues of Theorem B will be proved. A special case of one of these (Theorem 3) is implied by Van Est's result.

One way af proving Theorem $B$ is to use the fact that the only Lie subgroups of $\mathfrak{L}^{+}$are: 1 . discrete groups; 2 . Abelian groups of all transformations with a given pair of fixed points (this includes the hyperbolic, parabolic and elliptic cases); 3. groups of all transformations which leave invariant a given pair of points in $E$ (these are mixed hyperbolic-elliptic groups); 4. groups of all transformations which leave invariant a given point in $E ; 5$. $\mathfrak{Q}+$ itself. We shall prove an analogue of this fact in order to generalize Theorem $\mathrm{B}$.

\section{The rigid motions of hyperbolic space.}

Let $D$ be the unit ball $\left\{\left(x_{1}, \ldots, x_{n}\right) \mid x_{1}^{2}+\ldots+x_{n}^{2}<1\right\}$ and $E$ its boundary. $D$ together with the line element

$$
d s^{2}=\frac{4\left(d x_{1}^{2}+\ldots+d x_{n}^{2}\right)}{\left(1-x_{1}^{2}-\ldots-x_{n}^{2}\right)^{2}}
$$

is a model of $n$-dimensional hyperbolic space (with sectional curvature $-1)$.

The geodesics and geodesic surfaces (of various dimensions), which we shall call $\mathrm{h}$-lines and h-planes, are the intersections with $D$ of circles and spheres orthogonal to $E$. The group of rigid motions is the same as the group of isogonal transformations of $D$. This group, which we denote by $\mathfrak{L}$, is the future-preserving half of the Lorentz group in $n+1$ variables. That is, $\mathfrak{L}$ consists of the $(n+1) \times(n+1)$ matrices which leave invariant the half-cone

$$
\left\{\left(y_{1}, \ldots, y_{n+1}\right) \mid y_{1}^{2}+\ldots,+y_{n}^{2}-y_{n+1}^{2}=0, y_{n+1}>0\right\} \text {. }
$$

and have determinant \pm 1 . The transformations with positive determinant form a subgroup $\mathfrak{L}^{+}$of index 2 in $\mathfrak{L}$. These are the orientationpreserving motions (and conformal transformations) of $D$. An element $\left(a_{i j}\right) \in \mathfrak{Q}$ acts in $D$ according to the formula

$$
x_{i}^{\prime}=\frac{a_{i n+1} \sum_{k} x_{k}^{2}+2 \sum_{k} a_{i k} x_{k}+a_{i n+1}}{\left(a_{n+1, n+1}-1\right) \sum_{k} x_{k}^{2}+2 \sum_{k} a_{n+1, k} x_{k}+\left(a_{n+1, n+1}+1\right)},
$$

where in all of the sums the index $k$ runs from 1 to $n$. This also defines an isogonal map of $\bar{R}^{n}$ (Euclidean space completed by a point at infinity) which maps the closed ball $\bar{D}$ (onto itself).

In the 2-dimensional case, the elements of $\mathfrak{Q}^{+}$are classified into elliptic, parabolic and hyperbolic types. We shall give a similar classification for $\mathfrak{L}$ in arbitrary dimension. 
Let $\mathbf{f} \in \mathfrak{L}$. Since $\mathbf{f}$ is a continuous map of $\bar{D}$ into itself, it has at least one fixed point in $\bar{D}$ (according to the Brouwer fixed point theorem). If there is a fixed point in $D$, we shall call $f$ elliptic. If there is exactly one fixed point $z$, and $z \in E$, we shall call $\mathbf{f}$ parabolic. If there are exactly two fixed points $z_{1}$ and $z_{2}$, and $z_{1}, z_{2} \in E$, we shall call $\mathrm{f}$ loxodromic. If there are three fixed points $z_{1}, z_{2}, z_{3}$ which are on $E$ then $\mathrm{f}$ must be elliptic. For if $\gamma$ is the circle determined by $z_{1}, z_{2}, z_{3}$, and $\pi$ is the h-plane, such that $\gamma=\bar{\pi} \cap E$, then $\mathrm{f}$ leaves $\pi$ invariant. In fact $\pi$ with its induced metric is a hyperbolic plane, and $f$ induces a rigid motion in $\pi$. But a rigid motion in $\pi$, which has three fixed points on the boundary $\gamma$, must be the identity. Thus, every point of $\pi$ is a fixed point of $f$.

Now suppose that $f$ is elliptic and $z$ is a fixed point in $D$. As $\mathfrak{Q}$ is transitive in $D$, there exists $g \in \mathfrak{L}$, so that $\mathbf{g}(z)=0$ (the origin). The transformation $\mathrm{gfg}^{-1}$ has 0 as a fixed point and therefore $\mathrm{gfg}^{-1}$ is an orthogonal transformation. It is represented in $\mathfrak{L}$ by a matrix of the following form (see (1)):

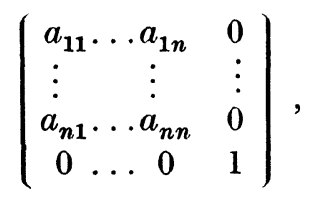

where $\left(a_{i j}\right)$ is an orthogonal matrix. By further conjugation, $f$ can be brought into the form

$$
\left(\begin{array}{lllll}
A_{1} & & & & \\
& A_{2} & & & \\
& & \ddots & & \\
& & & A_{\left[\frac{1}{2} n\right]} & \\
& & & & 1
\end{array}\right),
$$

where

$$
\begin{aligned}
{\left[\frac{1}{2} n\right] } & = \begin{cases}\frac{1}{2} n & \text { if } \quad n \text { is even, } \\
\frac{1}{2}(n+1) & \text { if } \quad n \text { is odd, }\end{cases} \\
A_{k} & =\left(\begin{array}{cc}
\cos \theta_{k} & \sin \theta_{k} \\
-\sin \theta_{k} & \cos \theta_{k}
\end{array}\right) \quad \text { if } \quad k<\frac{1}{2}(n+1),
\end{aligned}
$$

and $A_{(n+1) / 2}= \pm 1$. We shall normalize the $\theta_{k}$ by the condition $-\pi<\theta_{k} \leqq \pi$ and we shall call these normalized numbers the angles of $f$. We note that $f$ is diagonalizable (over the complex field) and its eigenvalues are $1, e^{ \pm i \theta_{k}}$ (where $\theta_{k}$ are the angles of $\mathbf{f}$ ) and possibly -1 .

If $S$ is a subset of $E$, let $\sigma[S]$ be the smallest sphere in $E$ which contains $S$, and let $\pi[S]$ be the h-plane so that $\sigma[S]=\overline{\pi[S]} \cap E$. If an orthogonal transformation leaves a set $S$ pointwise fixed, then it leaves the smallest 
plane containing $S$ pointwise fixed. From this it follows that if $S \subset E$, and $S$ is pointwise fixed under an elliptic transformation, then the same is true of $\sigma[S]$ and $\pi[S]$.

Now we shall consider the parabolic case. Let $\mathfrak{Q}^{\prime}$ be the group of isogonal transformations of $\bar{R}^{n}$. This group $\mathfrak{Q}^{\prime}$ is the future-preserving half of the Lorentz group in $n+2$ variables. An element $\left(a_{i j}\right) \in \mathfrak{R}^{\prime}$ acts in $\bar{R}^{n}$ according to the formula

$$
x_{i}^{\prime}=\frac{\left(a_{i n+2}+a_{i n+1}\right) \sum_{k} x_{k}^{2}+2 \sum_{k} a_{i k} x_{k}+\left(a_{i n+2}-a_{i n+1}\right)}{a \sum_{k} x_{k}^{2}+2 \sum_{k}\left(a_{n+2}-a_{n+1 k}\right) x_{k}+b},
$$

where $k$ runs from 1 to $n$ and

$$
\begin{aligned}
& a=a_{n+2, n+2}-a_{n+1, n+2}+a_{n+2, n+1}-a_{n+1, n+1}, \\
& b=a_{n+2, n+2}-a_{n+1, n+2}-a_{n+2, n+1}+a_{n+1, n+1} .
\end{aligned}
$$

A transformation in $\mathfrak{L}^{\prime}$ leaves $D$ invariant if and only if it has the form

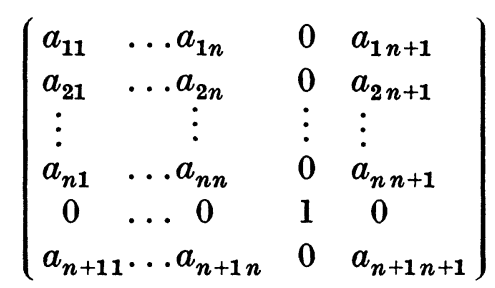

and a transformation $\left(a_{i j}\right) \in \mathfrak{L}$ is represented in $\mathfrak{L}^{\prime}$ by this matrix.

Now consider a parabolic transformation $\mathbf{f} \in \mathfrak{L}$, and let $z$ be the unique fixed point of $\mathbf{f}$. There is a transformation $\mathbf{g} \in \mathfrak{L}^{\prime}$ so that $\mathbf{g}(z)=\infty$ (for instance $\mathbf{g}=\mathbf{r}$, the reflection in some sphere with center $z$ ). Then $\mathbf{g f g}^{-1}$ is an isogonal transformation of Euclidean space, whose only fixed point is the point at infinity. Therefore

$$
\operatorname{gfg}^{-1}(x)=\varrho \mathbf{A} x+a,
$$

where $\varrho>0$ and $\mathbf{A}$ is an orthogonal transformation. Since $\operatorname{gfg}^{-1}(x)=x$ has no finite solution, $(1-\varrho \mathbf{A}) x=a$ has no solution, and $1-\varrho \mathbf{A}$ is a singular matrix. Therefore $\varrho^{-1}$ is an eigenvalue of $\mathbf{A}$. Since $\varrho>0$ and $\mathbf{A}$ is orthogonal, it follows that $\varrho=1$, and

$$
\operatorname{gfg}^{-1}(x)=\mathbf{A} x+a .
$$

Thus gfg $^{-1}$ is a screw-motion of Euclidean space. As such, it is conjugate to a transformation te, where $t$ is a translation in the $x_{n}$-direction, $\mathrm{e}$ is 
an orthogonal transformation with the origin as fixed point, and te =et. Thus $\mathbf{f}$ is conjugate to a transformation of the form

$$
\begin{aligned}
x^{\prime} & =\sum_{j=1}^{n-1} a_{i j} x_{j}, \quad 1 \leqq i \leqq n-1, \\
x^{\prime}{ }_{n} & =x_{n}+a,
\end{aligned}
$$

where $\left(a_{i j}\right)$ is an orthogonal matrix. This transformation is represented in $\mathfrak{L}^{\prime}$ by the following matrix (see (2)):

$$
\left(\begin{array}{ccccc}
a_{11} & \ldots a_{1 n-1} & 0 & 0 & 0 \\
\vdots & \vdots & \vdots & \vdots & \vdots \\
a_{n-11} \ldots a_{n-1 n-1} & 0 & 0 & 0 \\
0 & \ldots 0 & 1 & -a & a \\
0 & \ldots 0 & a & 1-\frac{1}{2} a^{2} & \frac{1}{2} a^{2} \\
0 & \ldots 0 & a & -\frac{1}{2} a^{2} & 1+\frac{1}{2} a^{2}
\end{array}\right)
$$

We shall refer to the angles of e also as the angles of $\mathbf{f}$. If $\mathbf{f}$ is conjugate to a translation, we shall call it strictly parabolic. In this case it is conjugate to a transformation with matrix

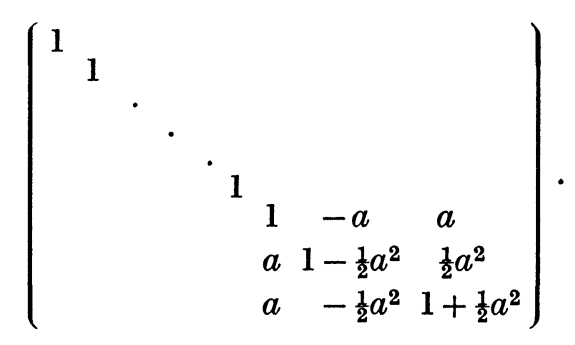

We note the following facts concerning a parabolic transformation. $f$ is not diagonalizable (over the complex field) and its eigenvalues are 1 (with multiplicity at least 3 ), $e^{ \pm i \theta_{k}}$ (where $\theta_{k}$ are the angles of $\mathbf{f}$ ) and possibly -1 . There is a unique strictly parabolic transformation $\mathbf{s}$, and a unique elliptic transformation $e$, so that $\mathbf{f}=\mathbf{s e}=\mathbf{e s}$. If $\mathbf{f}$ is parabolic, it is strictly parabolic if and only if its only eigenvalue is 1 .

Now suppose $\mathbf{f}$ is loxodromic and $z_{1}$ and $z_{2}$ are its fixed points. There is a transformation $\mathbf{g} \in \mathfrak{L}^{\prime}$, so that $\mathbf{g}\left(z_{1}\right)=0$ and $\mathbf{g}\left(z_{2}\right)=\infty$ (for instance $\mathbf{g}=\operatorname{tr}$, where $\mathbf{r}\left(z_{2}\right)=\infty$ and $\mathbf{t}$ is a translation such that $\operatorname{tr}\left(z_{1}\right)=0$ ). $\mathbf{g f g}^{-1}$ is an isogonal transformation whose only fixed points are 0 and $\infty$. It follows that $\mathrm{gfg}^{-1}=\mathrm{he}$, where $\mathrm{h}$ is a dilatation $(\mathrm{h}(x)=\varrho x, \varrho>0, \varrho \neq 1)$ and e is an orthogonal transformation with 0 as fixed point. If $\varrho=e^{\lambda}, \mathbf{g f g}^{-1}$ is represented by the following matrix (see (2)): 


$$
\left(\begin{array}{cccc}
a_{11} \ldots & a_{1 n} & 0 & 0 \\
\vdots & \vdots & \vdots & \vdots \\
a_{n 1} \ldots & a_{n n} & 0 & 0 \\
0 \ldots & \ldots & \cosh \lambda & \sinh \lambda \\
0 \ldots & \ldots & \sinh \lambda & \cosh \lambda
\end{array}\right)
$$

where $\left(a_{i j}\right)$ is an orthogonal matrix. We shall refer to the angles of $\left(a_{i j}\right)$ also as the angles of $f$. If $f$ is conjugate to a dilatation, we shall call it hyperbolic. In this case, it is conjugate to a transformation with matrix

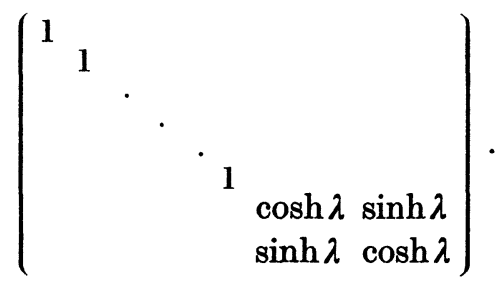

We note the following facts concerning a loxodromic transformation. The $\mathrm{h}$-line between the fixed points of $\mathrm{f}$ is invariant. We shall call this h-line the axis of $\mathbf{f}$, and it will be denoted $A_{\mathrm{f}}$. Points on $A_{\mathrm{f}}$ are translated a constant distance $\lambda$ (which is the same number that appears in (3)). This distance will be called the translation length of $\mathbf{f}$. The transformation $f$ is diagonalizable (over the complex field) with eigenvalues $e^{ \pm \lambda}$, $e^{ \pm i \theta_{k}}$ (where $\lambda$ is the translation length and $\theta_{k}$ are the angles of $f$ ) and possibly -1 . There is a unique hyperbolic transformation $h$ and $a$ unique elliptic transformation $e$, so that $f=h e=h$. If $f$ is loxodromic, it is hyperbolic if and only if 1 is an eigenvalue with multiplicity $n-1$.

We shall summarize these facts in the following propositions. By diagonalizable we shall mean diagonalizable over the complex field.

Proposition 1.

1) If $\mathbf{f}$ is hyperbolic, then it is diagonalizable, it has eigenvalues 1 (with multiplicity $n-1)$ and $e^{ \pm \lambda}$ (where $\lambda$ is the translation length of $\mathbf{f}$ ), and it is conjugate (in $\mathfrak{L}^{\prime}$ ) to a dilatation.

2) If $\mathbf{f}$ is elliptic, then it is diagonalizable, its eigenvalues are $1, e^{ \pm i \theta_{k}}$ (where $\theta_{k}$ are the angles of $\mathbf{f}$ ) and possibly -1 , and it is conjugate (in $\mathfrak{L}$ ) to an orthogonal transformation.

3) If $\mathbf{f}$ is strictly parabolic, then its only eigenvalue is 1 , and it is conjugate (in $\left.\mathbf{\Omega}^{\prime}\right)$ to a translation of Euclidean space.

4) If $\mathbf{f}$ is loxodromic, then it is diagonalizable, it has eigenvalues $e^{ \pm \lambda}$, $e^{ \pm i \theta_{k}}$ (where $\lambda$ is the translation length and $\theta_{k}$ are the angles of $\mathbf{f}$ ) and possibly -1 , and it is conjugate (in $\left.\mathfrak{L}^{\prime}\right)$ to the product of a dilatation and an orthogonal transformation (with a common fixed point). There is a unique hyper- 
bolic transformation $\mathbf{h}$ and $a$ unique elliptic transformation $\mathbf{e}$, so that $\mathbf{f}=$ he $=$ eh.

5) If $\mathbf{f}$ is parabolic, then it is not diagonalizable, its eigenvalues are 1 (with multiplicity at least 3 ), $e^{ \pm i \theta_{k}}$ (where $\theta_{k}$ are the angles of f) and possibly -1, and it is conjugate (in $\mathfrak{R}^{\prime}$ ) to a screw-motion of Euclidean space. There is a unique strictly parabolic transformation $\mathrm{s}$ and a unique elliptic transformation $\mathbf{e}$, so that $\mathbf{f}=\mathbf{s e}=\mathbf{e s}$.

Proposition 2. Let $S$ be a subset of $E$ which contains more than two points and which is pointwise fixed by a transformation $\mathbf{f} \in \mathfrak{R}$. Then $\sigma[S]$ and $\pi[S]$ are pointwise fixed by $\mathbf{f}$.

We shall conclude this section by remarking that Nielsen's theorem (Theorem A) is true in all dimensions. This fact follows from Nielsen's theorem and the following.

Proposition 3. A hyperbolic group $\mathfrak{H}$ has an invariant circle in $E$, which contains all fixed points of elements in $\mathfrak{\mathfrak { S }}$.

Proof. If all elements of $\mathfrak{W}$ have the same pair of fixed points, the assertion is clear (for any circle through the fixed points). Otherwise, let $\mathbf{h}_{\mathbf{1}}, \mathbf{h}_{\mathbf{2}} \in \mathfrak{H}$ and suppose their fixed points do not coincide. Let $\sigma$ be the (1- or 2-dimensional) sphere which contains these fixed points. The hype bolic group $\left\langle\mathbf{h}_{1}, \mathbf{h}_{2}\right\rangle$ leaves $\sigma$ invariant, and $\sigma$ contains all fixed points of elements in this group. This is true because a sphere $\sigma$ is invariant under a hyperbolic transformation $\mathbf{h}$, if and only if $\sigma$ contains the fixed points of $\mathbf{h}$. Van Vleck [6] has shown that a hyperbolic group of linear fractional transformations has an invariant circle. Therefore there is a circle $\gamma$ which contains the fixed points of all elements in $\left\langle\mathbf{h}_{1}, \mathbf{h}_{2}\right\rangle$. We shall show that if $\mathbf{h} \in \mathfrak{S}$, the fixed points of $\mathbf{h}$ are on $\gamma$. By the above reasoning, the fixed points of the group $\left\langle h_{1}, \mathbf{h}\right\rangle$ lie on a circle $\gamma^{\prime}$, which intersects $\gamma$ (at least) in the fixed points of $h_{1}$. Let $\sigma^{\prime}$ be the sphere containing $\gamma$ and $\gamma^{\prime}$. The group $\left\langle\mathbf{h}_{1}, \mathbf{h}_{2}, \mathbf{h}\right\rangle$ leaves $\sigma^{\prime}$ invariant, so by Van Vleck's theorem there is an invariant circle. This circle must be $\gamma$. Thus all fixed points of $\mathfrak{S}$ are on $\gamma$.

\section{The Lie algebra.}

Let $\mathscr{L}$ be the Lie algebra of $\mathfrak{L}$.

The representation of $\mathscr{L}$ as skew-symmetric tensors and the consequent easy derivation of the Killing form can be found in [5]. It is included here for convenience.

Since $\mathfrak{L}$ contains (with index 2 ) the connected component of the group of linear transformations which leave invariant the form 


$$
(x, y)=x_{1} y_{1}+\ldots+x_{n} y_{n}-x_{n+1} y_{n+1},
$$

$\mathscr{L}$ consists of the linear transformations which are skew-symmetric with respect to this form. That is, $X \in \mathscr{L}$ if and only if $(X u, v)=-(u, X v)$. The mappings $\Phi(v): u \rightarrow(u, v)$ define an isomorphism $\Phi$ of the vector space $V$ onto the dual space $V^{*}$. If $X$ is any linear transformation of $V$ and $X^{\top}$ is the dual transformation, then $X^{*}=\Phi^{-1} X^{\top} \Phi$ is the linear transformation of $V$, such that $(X u, v)=\left(u, X^{*} v\right)$. The Lie algebra $\mathscr{L}$ consists of those $X$ such that $X^{*}=-X$. The isomorphisme $\Phi$ also defines an isomorphism of $V \otimes V$ onto $V \otimes V^{*}$. The latter is canonically isomorphic to the Lie algebra $\mathscr{G}$ of all linear transformations of $V$. Thus $V \otimes V$ is isomorphic to $\mathscr{G}$, and an element $X=x_{1} \otimes x_{2} \in V \otimes V$ operates as a linear transformation of $V$ as follows:

$$
X u=x_{1}\left(x_{2}, u\right) \text {. }
$$

Hence $(X u, v)=\left(x_{1}, v\right)\left(x_{2}, u\right)=\left(u, X^{*} v\right)$, so that $X^{*} v=x_{2}\left(x_{1}, v\right)$. Thus the mapping $X \rightarrow X^{*}$ corresponds to the symmetry $S$ in $V \otimes V$, and $\mathscr{L}$ corresponds to the space $\mathscr{S}$ of skew-symmetric tensors in $V \otimes V$. If $X=x_{1} \otimes x_{2}, Y=y_{1} \otimes y_{2}$, then

$$
\begin{aligned}
{[X Y]=X Y-Y X } & =\left(x_{2}, y_{1}\right) x_{1} \otimes y_{2}-\left(y_{2}, x_{1}\right) y_{1} \otimes x_{2} \\
& =\left(X \otimes 1-1 \otimes X^{*}\right) y_{1} \otimes y_{2} .
\end{aligned}
$$

Thus the operator ad $X: Y \rightarrow[X Y]$ corresponds to the operator $X \otimes 1-$ $1 \otimes X^{*}$ in $V \otimes V$. If $X \in \mathscr{L}$, ad $X$ (in $\mathscr{L}$ ) corresponds to $X \otimes 1+1 \otimes X$ (restricted to $\mathscr{S}$ ).

Proposition 4. Let $X \in \mathscr{L}$.

a) If $X$ is diagonalizable, then $\operatorname{ad} X$ is also, and the eigenvalues of $\operatorname{ad} X$ are $\left\{\lambda_{i}+\lambda_{j} \mid i \neq j\right\}$, where $\left\{\lambda_{k} \mid k=1, \ldots, n+1\right\}$ are the eigenvalues of $X$ (each listed the number of times equal to its multiplicity).

b) If $X$ is nilpotent, then ad $X$ is nilpotent.

Proof. By the above remarks, we can substitute $X \otimes 1+1 \otimes X$ for $\operatorname{ad} X$ and $\mathscr{S}$ for $\mathscr{L}$.

a) Let $v_{1}, \ldots, v_{n}$ be a basis of eigenvectors in the complexification of $V$. If $X v_{i}=\lambda_{i} v_{i}$, then

$$
(X \otimes 1+1 \otimes X)\left(v_{i} \otimes v_{j}-v_{j} \otimes v_{i}\right)=\left(\lambda_{i}+\lambda_{j}\right)\left(v_{i} \otimes v_{j}-v_{j} \otimes v_{i}\right) .
$$

b) Suppose $X^{N}=0$. Then

$$
(X \otimes 1+1 \otimes X)^{2 N}=\sum_{k=0}^{2 N}\left(\begin{array}{c}
2 N \\
k
\end{array}\right) X^{k} \otimes X^{2 N-k}=0 .
$$


For later use, we will now compute the Killing form $\langle X, Y\rangle=$ $\operatorname{tr}(\operatorname{ad} X$, ad $Y)$. Let $S$ be the symmetry in $V \otimes V$, that is, $S(x \otimes y)=$ $y \otimes x)$, and $\mathscr{S}=\{w \in V \otimes V \mid S(w)=-w\}$. The operator $\frac{1}{2}(1-S)$ is a projection of $V \otimes V$ onto $\mathscr{S}$. Thus

$$
\begin{aligned}
\langle X, X\rangle= & \operatorname{tr} \mathscr{S}\left\{(X \otimes 1+1 \otimes X)^{2}\right\} \\
= & \operatorname{tr}\left\{\frac{1}{2}(1-S)(X \otimes 1+1 \otimes X)^{2}\right\} \\
= & \frac{1}{2}\left\{2(n+1) \operatorname{tr}\left(X^{2}\right)+2 \operatorname{tr}(X)^{2}\right\}- \\
& -\frac{1}{2}\left\{\operatorname{tr}\left(S\left(X^{2} \otimes 1\right)\right)+2 \operatorname{tr}(S(X \otimes X))+\operatorname{tr}\left(S\left(1 \otimes X^{2}\right)\right)\right\} .
\end{aligned}
$$

But $\operatorname{tr}\{S(A \otimes B)\}=\operatorname{tr}(A B)$. For if $A=a_{1} \otimes a_{2}$ and $B=b_{1} \otimes b_{2}$, then

$$
\begin{aligned}
A B=a_{1} \otimes b_{2}\left(a_{2}, b_{1}\right), \quad & \operatorname{tr}(A B)=\left(a_{1}, b_{2}\right)\left(a_{2}, b_{1}\right), \\
(A \otimes B)(x \otimes y)=A x \otimes B y & =a_{1} \otimes b_{1}\left(a_{2}, x\right)\left(b_{2}, y\right) \\
& =\left[\left(a_{1} \otimes b_{1}\right) \otimes\left(a_{2} \otimes b_{2}\right)\right](x \otimes y) .
\end{aligned}
$$

Thus $A \otimes B=\left(a_{1} \otimes b_{1}\right) \otimes\left(a_{2} \otimes b_{2}\right)$ and

$$
\begin{aligned}
& S(A \otimes B)=\left(S\left(a_{1} \otimes b_{1}\right)\right) \otimes\left(a_{2} \otimes b_{2}\right)=\left(b_{1} \otimes a_{1}\right) \otimes\left(a_{2} \otimes b_{2}\right), \\
& \operatorname{tr}\{S(A \otimes B)\}=\left(b_{1} \otimes a_{1}, a_{2} \otimes b_{2}\right)=\left(b_{1}, a_{2}\right)\left(a_{1}, b_{2}\right)=\operatorname{tr}(A B) .
\end{aligned}
$$

Therefore

$$
\langle X, X\rangle=(n-1) \operatorname{tr}\left(X^{2}\right)+\operatorname{tr}(X)^{2} .
$$

But $X^{*}=\Phi^{-1} X^{\top} \Phi$ implies $\operatorname{tr} X^{*}=\operatorname{tr} X$, and $X^{*}=-X$ implies $\operatorname{tr} X^{*}=$ $-\operatorname{tr} X$. Therefore

$$
\operatorname{tr} X=0 \quad \text { and } \quad\langle X, X\rangle=(n-1) \operatorname{tr}\left(X^{2}\right) .
$$

The following proposition is a consequence of the above discussion and Proposition 1.

Proposition 5. Let $X \in \mathscr{L}$.

a) If $\exp X$ is elliptic or parabolic, then $\langle X, X\rangle=-2(n-1) \Sigma \theta_{k}^{2}$, where the $\theta_{k}$ are congruent $\bmod 2 \pi$ to the angles of $\exp X$.

b) If $\exp X$ is loxodromic, then $\langle X, X\rangle=2(n-1)\left[\lambda^{2}-\Sigma \theta_{k}^{2}\right]$, where $\lambda$ is the translation length and the $\theta_{k}$ are congruent $\bmod 2 \pi$ to the angles of $\exp X$.

The following proposition is proved in [1].

Proposition 6. Let $\mathscr{R}$ be a real Lie algebra and let $\mathbf{A}$ be an automorphism of $\mathscr{R}$. Then there exist derivations $\Delta_{1}, \Delta_{3}$ and an automorphism $\mathbf{A}_{2}$ so that

1) $\mathbf{A}=\exp \Delta_{1} \cdot \mathbf{A}_{2} \cdot \exp \Delta_{3}$.

2) $\Delta_{1}$ is diagonalizable with real eigenvalues; $\mathbf{A}_{2}$ is diagonalizable with eigenvalues of absolute value $1 ; \Delta_{3}$ is nilpotent. 
3) $\Delta_{1}, \mathbf{A}_{2}$ and $\Delta_{3}$ commute with any linear transformation that commutes with $\mathbf{A}$.

Furthermore, if $\Delta_{1}{ }^{\prime}, \Delta_{3}{ }^{\prime}$ are derivations and $\mathbf{A}_{2}{ }^{\prime}$ an automorphism such that 1) and 2) are satisfied and $\exp \Delta_{2}{ }^{\prime}, \mathbf{A}_{2}{ }^{\prime}, \exp \Delta_{3}{ }^{\prime}$ mutually commute, then $\Delta_{1}{ }^{\prime}=\Delta_{1}, \mathbf{A}_{2}{ }^{\prime}=\mathbf{A}_{2}$ and $\Delta_{3}{ }^{\prime}=\Delta_{3}$.

We shall refer to the above decomposition as the canonical decomposition of $\mathbf{A}$.

For each element $\mathbf{a} \in \mathfrak{L}$, the mapping $\mathbf{f} \rightarrow \mathrm{afa}^{-1}$ induces an automorphism $\mathbf{A}$ of $\mathscr{L}$. We shall denote the group of these automorphisms by $\mathfrak{A}_{\mathfrak{L}}$. Since $\mathfrak{L}^{+}$has trivial centralizer in $\mathfrak{L}$, the mapping $\mathbf{a} \rightarrow \mathbf{A}$ is an isomorphism.

Proposition 7. Let $\mathbf{a} \in \mathfrak{L}$ and let $\mathbf{A}$ be the corresponding automorphism of $\mathscr{L}$.

1) a is hyperbolic if and only if $\mathrm{A}=\exp (\operatorname{ad} X)$, where $X \in \mathscr{L}$ and $\operatorname{ad} X$ is diagonalizable with real eigenvalues.

2) $\mathbf{a}$ is elliptic if and only if $\mathbf{A}$ is diagonalizable with eigenvalues of absolute value 1 .

3) $\mathbf{a}$ is strictly parabolic if and only if $\mathbf{A}=\exp (\operatorname{ad} X)$, where $X \in \mathscr{L}$ and ad $X$ is nilpotent.

4) $\mathbf{a}$ is loxodromic if and only if $\mathbf{A}=\exp (\operatorname{ad} X) \cdot \mathbf{B}=\mathbf{B} \cdot \exp (\operatorname{ad} X)$, where $X \in \mathscr{L}$, ad $X$ is diagonalizable with real eigenvalues, $\mathbf{B} \in \mathfrak{A}_{\mathfrak{L}}$ and $\mathbf{B}$ is diagonalizable with eigenvalues of absolute value 1.

5) $\mathbf{a}$ is parabolic if and only if $\mathbf{A}=\exp (\operatorname{ad} X) \cdot \mathbf{B}=\mathbf{B} \cdot \exp (\operatorname{ad} X)$, where $X \in \mathscr{L}, \operatorname{ad} X$ is nilpotent, $\mathbf{B} \in \mathfrak{A}_{\mathfrak{L}}$ and $\mathbf{B}$ is diagonalizable with eigenvalues of absolute value 1 .

Proof. The uniqueness in Proposition 6 shows that the above alternatives concerning $\mathbf{A}$ are mutually exclusive. Therefore, it is enough to prove only if in each case.

1) Suppose that $\mathbf{a}$ is hyperbolic. Then there is a matrix $\mathbf{m}$, so that $\operatorname{mam}^{-1}=\operatorname{diag}\left(1, \ldots, 1, e^{\lambda}, e^{-\lambda}\right)$, where $\lambda$ is real. Define $X=\mathbf{m}^{-1} \operatorname{diag}(0, \ldots$, $0, \lambda,-\lambda) \mathrm{m}$. Then $X \in \mathscr{L}$ and $\mathbf{a}=\exp X$. The isomorphism $\mathfrak{L} \rightarrow \mathfrak{A}_{\mathfrak{L}}$ induces an isomorphism of the Lie algebras, which is given by $X \rightarrow \operatorname{ad} X$. Therefore $A=\exp (\operatorname{ad} X)$. Proposition 4 shows that ad $X$ is diagonalizable with real eigenvalues.

2) Suppose a is elliptic. Let $z$ be any fixed point of $\mathbf{a}$ in $D$, and let $\Re$ be the subgroub of transformations which leave $z$ fixed. $\Re$ is isomorphic to the orthogonal group. Therefore any representation of $\mathbb{R}$ (in particular $\mathfrak{R} \rightarrow \mathfrak{A}_{\mathfrak{\Omega}}$ ) is equivalent to a representation by orthogonal transformations. Hence $\mathbf{A}$ is diagonalizable with eigenvalues of absolute value 1 . 
3) Suppose a is strictly parabolic. Then $1-a$ is nilpotent and the series

$$
X=\log (\mathbf{1}-(\mathbf{1}-\mathbf{a}))=-(\mathbf{1}-\mathbf{a})-\frac{1}{2}(\mathbf{1}-\mathbf{a})^{2}-\ldots
$$

converges. $X \in \mathscr{L}, \mathbf{a}=\exp X$ and $X$ is nilpotent. Proposition 4 shows that $\operatorname{ad} X$ is nilpotent. As in 1$), A=\exp (\operatorname{ad} X)$.

4) If $a$ is loxodromic, then $a=a_{1} b=b a_{1}$, where $a_{1}$ is hyperbolic and $b$ is elliptic. If $A_{1}$ and $B$ are the automorphisms corresponding to $a_{1}$ and $b$, then $\mathbf{A}=\mathbf{A}_{1} \mathbf{B}=\mathbf{B} \mathbf{A}_{1}$. Cases 1) and 2) imply that $\mathbf{A}_{1}=\exp (\operatorname{ad} X)$, where $\operatorname{ad} X$ is diagonalizable with real eigenvalues, and $\mathbf{B}$ is diagonalizable with eigenvalues of absolute value 1 .

5) If $a$ is parabolic, then $a=a_{1} b=b a_{1}$, where $a_{1}$ is strictly parabolic and $b$ is elliptic. The assertion follows as in 4).

If $\mathbb{B S}$ is a connected, semi-simple Lie subgroup of $\mathfrak{Q}$ with trivial center, and $\mathscr{G}$ is the Lie algebra of $\mathscr{H}$, then there are the natural inclusions $\mathscr{G} \subset \mathscr{L}$ and $\mathfrak{A}_{\mathfrak{G}} \subset \mathfrak{A}_{\mathfrak{L}}$. Suppose $\mathbf{a} \in \mathbb{S}, \mathbf{A}$ is the corresponding automorphism in $\mathfrak{A}_{\mathfrak{G}}$, and $\mathbf{A}=\exp \Delta_{1} \cdot \mathbf{A}_{2} \cdot \exp \Delta_{3}$ is the canonical decomposition of $\mathbf{A}$ relative to $\mathscr{G}$. Since $\mathfrak{B S}^{\mathrm{S}}$ is connected and semi-simple, $\mathfrak{A}_{\mathfrak{G}}$ is the component of the identity in the group of automorphisms of $\mathscr{G}$. Therefore $\exp \Delta_{1}$ and $\exp \Delta_{3}$ are in $\mathfrak{A}_{\mathfrak{B}}$, so that $\mathbf{A}_{2}$ is also in $\mathfrak{A}_{\mathfrak{G}}$. Thus, all of these transformations can be considered as acting in $\mathscr{L}$. Note also that since $\mathscr{G}$ is semi-simple there exist elements $X_{1}, X_{3} \in \mathscr{G}$, so that $\Delta_{1}=\operatorname{ad} X_{1}$ and $\Delta_{3}=\operatorname{ad} X_{3}$. Thus we have $\mathbf{A}=\exp \left(\operatorname{ad} X_{1}\right) \cdot \mathbf{A}_{2} \cdot \exp \left(\operatorname{ad} X_{3}\right)$. On the other hand we have the decomposition relative to $\mathscr{L}$

$$
\mathbf{A}=\exp \left(\operatorname{ad} X_{1}{ }^{\prime}\right) \cdot \mathbf{A}_{2}{ }^{\prime} \cdot \exp \left(\operatorname{ad} X_{3}{ }^{\prime}\right) .
$$

Proposition 8. If \&S is a connected, semi-simple Lie subgroup of $\mathbb{2}$ with trivial centralizer, then $X_{1}{ }^{\prime}=X_{1}, \mathbf{A}_{2}{ }^{\prime}=\mathbf{A}_{2}$ and $X_{3}{ }^{\prime}=X_{3}$.

Proof. Since $\operatorname{ad} X_{1}{ }^{\prime}, \mathbf{A}_{2}{ }^{\prime}$ and ad $X_{3}{ }^{\prime}$ commute with any linear transformation that commutes with $\mathbf{A}$, it follows that these transformations are polynomials in A. Therefore $\operatorname{ad} X_{1}{ }^{\prime}, \mathbf{A}_{2}{ }^{\prime}$ and $\operatorname{ad} X_{3}{ }^{\prime}$ leave $\mathscr{G}$ invariant. Because of the uniqueness of the decomposition relative to $\mathscr{G}$, we have for the restrictions to $\mathscr{G}$

$$
\begin{aligned}
\operatorname{ad} X_{1}^{\prime} \mid \mathscr{G} & =\operatorname{ad} X_{1} \mid \mathscr{G}, \\
\mathbf{A}_{2}^{\prime} \mid \mathscr{G} & =\mathbf{A}_{2} \mid \mathscr{G}, \\
\operatorname{ad} X_{3}^{\prime} \mid \mathscr{G} & =\operatorname{ad} X_{2} \mid \mathscr{G} .
\end{aligned}
$$

Therefore $X_{1}^{\prime}-X_{1}$ and $X_{3}{ }^{\prime}-X_{3}$ are in the centralizer of $\mathscr{G}$. Since the centralizer is trivial, $X_{1}{ }^{\prime}=X_{1}$ and $X_{3}{ }^{\prime}=X_{3}$. It now also follows that $\mathbf{A}_{2}^{\prime}=\mathbf{A}_{2}$. 
Proposition 9. Let (S) be a connected, semi-simple Lie subgroup of $\mathfrak{L}$ with trivial centralizer. Then 18 contains the hyperbolic, elliptic and strictly parabolic components of its elements. The hyperbolic and strictly parabolic elements are contained in (hyperbolic and strictly parabolic) 1-parameter subgroups of $\mathbb{B S}$.

Proof. Suppose that a is a loxodromic (parabolic) element of $\mathbb{E S . ~ T h e n ~}$ $a=a_{1} a_{2}=a_{2} a_{1}$, where $a_{1}$ is hyperbolic (strictly parabolic) and $a_{2}$ is elliptic. Let $\mathbf{A}, \mathbf{A}_{1}, \mathbf{A}_{2}$ be the corresponding automorphisms. Then $\mathbf{A}=\mathbf{A}_{\mathbf{1}} \mathbf{A}_{\mathbf{2}}=$ $\mathbf{A}_{\mathbf{2}} \mathbf{A}_{1}$. Proposition 7 implies that this is the canonical decomposition of $\mathbf{A}$ (relative to $\mathscr{L}$ ). Proposition 8 implies that the components $\mathbf{A}_{1}$ and $\mathbf{A}_{2}$ are in $\mathfrak{A}_{\mathscr{G}}$. Therefore $\mathbf{a}_{1}$ and $\mathbf{a}_{2}$ are elements of $\mathbb{A S}$.

As we have seen, $\mathbf{A}_{1}=\exp (\operatorname{ad} X)$, where $X \in \mathscr{L}$, and $\operatorname{ad} X$ is diagonalizable with real eigenvalues, or $\operatorname{ad} X$ is nilpotent. Therefore $a_{1}$ lies on a hyperbolic or strictly parabolic 1-parameter subgroup of $\mathbb{5 5}$.

Proposition 10. Let (S) be a connected Lie group with trivial center, and let $\subseteq$ be a connected, semi-simple Lie subgroup with trivial centralizer. Then $\subseteq$ is a closed subgroup of $\mathbb{S}$.

Proof. Let $\mathscr{G}$ and $\mathscr{S}$ be the Lie algebras of $\mathscr{S}$ and $\mathfrak{S}$, respectively. The mapping $\mathscr{S} \rightarrow \mathfrak{A}_{\mathscr{G}}$ is an isomorphism and homeomorphism. Let $\mathfrak{B}$ be the subroup of $\mathfrak{A}_{\mathfrak{G}}$ which leaves $\mathscr{S}$ invariant. $\mathfrak{B}$ is a closed subgroup of $\mathfrak{A}_{\mathfrak{G}}$. Each element of $\mathfrak{B}$ induces an automorphism of $\mathscr{S}$, and we have a homomorphism $\mathfrak{B} \rightarrow \operatorname{Aut}(\mathscr{S})$. In fact this is an isomorphism, since the centralizer of $\mathfrak{S}$ is trivial. Since $\mathfrak{S}$ is semi-simple, $\mathfrak{A}_{\mathfrak{S}}$ is the component of the identity in $\operatorname{Aut}(\mathscr{S})$, and $\mathfrak{A}_{\overparen{\subseteq}}$ is a closed subgroup of $\operatorname{Aut}(\mathscr{S})$. Thus $\mathfrak{A}_{\overparen{\subseteq}}$ is closed in $\mathfrak{B}$, and $\mathfrak{B}$ is closed in $\mathfrak{A}_{\mathfrak{G}}$. Therefore $\mathfrak{A}_{\mathfrak{S}}$ is closed in $\mathfrak{A}_{\mathfrak{S}}$ and $\mathfrak{S}$ is closed in $\mathfrak{G}$.

\section{The Lie subgroups of $\mathfrak{L}$.}

Let $\mathbb{S S}$ be a subgroup of $\mathfrak{L}$. The set of limit points of $\mathbb{S}$, denoted $L_{\mathfrak{G}}$, is defined to be the intersection with $E$ of the limit set of any orbit in $D$. That is,

$$
L_{\mathscr{S}}=E \cap\{\mathbf{g}(z) \mid \mathbf{g} \in \mathbb{B S}\}^{\prime},
$$

where $z$ is an arbitrary point in $D$. This set is independent of $z$ because hyperbolic distances (which are preserved by (S) become arbitrarily small relative to Euclidean distance as $E$ is approached. $L_{\mathfrak{G}}$ is a closed subset of $E$ which is invariant under $\mathbb{B S}$. It is easy to see that if $\overline{\mathbb{S}}$ is the closure of $\mathfrak{G}$, then $L_{\overparen{S}}=L_{\mathfrak{S}}$.

The Lie subgroups of $\mathfrak{Q}$ are conveniently divided into four classes, according as $L_{\mathscr{S}}$ is empty, contains exactly one point, contains exactly 
two points or contains more than two points. If the elements of $\mathbb{S}$ have a common fixed point $z \in E$, and $\mathrm{f}$ is an isogonal transformation of $\bar{R}^{\boldsymbol{n}}$ so that $\mathbf{f}(z)=\infty$, then $\mathbf{f S S f}^{-1}$ leaves $\infty$ fixed. Thus $\mathbb{B S}$ is conjugate to a group of isogonal transformations of Euclidean space. If $L_{\leftrightarrow}$ consists of one point, then this point is fixed under (B) and (5) contains only elliptic and parabolic transformations. In this case, (S) is conjugate to a group of rigid motions of Euclidean space. We shall not investigate groups of these types any further. In the following we shall take a closer look at those Lie subgroups $\mathbb{B S}$ of $\mathfrak{Q}$ such that $\mathbb{B S}$ does not have a common fixed point in $E$, and $L_{\text {लs }}$ is empty or contains at least two points. We shall first obtain some more information about $L_{\mathfrak{\leftrightarrow}}$.

Proposition 11. If $S$ is a closed subset of $E$ which contains more than one point, and $S$ is invariant under $\mathbb{S}$, then $S \supset L_{\mathfrak{\leftrightarrow}}$.

Proof. Let $z \in L_{\mathscr{B}}$ and $z_{1}, z_{2} \in S$. There is a sequence $\left\{\mathbf{g}_{n}\right\} \subset$ \&S so that $\lim \mathrm{g}_{n}(p)=z$ for any $p \in D$. We assert that at least one of the sequences $\left\{\mathbf{g}_{n}\left(z_{1}\right)\right\},\left\{\mathbf{g}_{n}\left(z_{2}\right)\right\}$ has $z$ as a limit point. If this is false, then there is a subsequence $\left\{\mathbf{g}_{n_{k}}\right\}$ so that $\lim _{k \rightarrow \infty} \mathbf{g}_{n_{k}}\left(z_{1}\right)=x_{1}$ and $\lim _{k \rightarrow \infty} \mathbf{g}_{n_{k}}\left(z_{2}\right)=x_{2}$, where $x_{1} \neq z$ and $x_{2} \neq z$ (though possibly $x_{1}=x_{2}$ ). Let $\lambda$ be the h-line with endpoints $z_{1}, z_{2}$ and let $p$ be a point on $\lambda$. There are neighborhoods $U_{1}$ of $x_{1}$ and $U_{2}$ of $x_{2}$ so that $z \notin \bar{U}_{1}$ and $z \notin \bar{U}_{2}$. For large $k, \mathbf{g}_{n_{k}}\left(z_{1}\right) \in U_{1}$ and $\mathbf{g}_{n_{k}}\left(z_{2}\right) \in U_{2}$, while $\mathbf{g}_{n_{k}}(p)$ is a point on the $\mathrm{h}$-line $\mathbf{g}_{n_{k}}(\lambda)$. If the sequence $\left\{\boldsymbol{g}_{n_{k}}(p)\right\}$ converges to a point of $E$, this point must be in $\bar{U}_{1}$ or $\bar{U}_{2}$. We have thereby reached a contradiction. Since $S$ is closed and invariant, the proposition now follows from the above assertion.

Lemma 1. Suppose that the elements of (S) do not have a common fixed point in $E$, and $\mathbf{f}$ is a loxodromic element of \&5 whose axis is not invariant under (S). If $U$ is an open subset of $E$ which contains a point of $L_{\mathfrak{G}}$, then there is an element $\mathbf{f}^{\prime}$ which is conjugate to $\mathbf{f}$ (in (S)) and has its fixed points in $U$.

Proof. Let $u$ and $v$ be the fixed points of $\mathrm{f}$. Let us assume that $u$ and $v$ are labelled so that $\lim _{n \rightarrow \infty} \mathrm{f}^{n}(z)=v$, if $z \neq u$, and $\lim _{n \rightarrow \infty} \mathrm{f}^{-n}(z)=u$, if $z \neq v$.

We shall first show that there is $\mathbf{g} \in \mathbb{S}$ so that $\mathbf{g}(u) \neq u, v$ and $\mathbf{g}(v) \neq u, v$. The assumption that the axis $A_{\mathrm{f}}$ is not invariant under (S) means that there is an element $\mathbf{h} \in \mathbb{E S}$, so that the pair of points $\{\mathbf{h}(u), \mathbf{h}(v)\}$ does not coincide with $\{u, v\}$, say $\mathbf{h}(v) \neq u, v$. Then $v=\lim _{n \rightarrow \infty} \mathbf{f}^{n} \mathbf{h}(v)$, and there are an infinite number of points in the orbit $\mathbb{S} v$. It now easily follows that the same is true of the orbit $\mathbb{S} u$ (for it is dense in $L_{\mathfrak{S}}$, which contains $g(v))$. Therefore there are elements $\mathbf{g}_{1}, \mathbf{g}_{2} \in G$, so that $\mathbf{g}_{1}(u) \neq u, v$, 
$\mathbf{g}_{2}(v) \neq u, v$ and $\mathbf{g}_{1}(u) \neq \mathbf{g}_{2}(v)$. If $\mathbf{g}_{1}(v) \neq u, v$ or $\mathbf{g}_{2}(u) \neq u, v$ then our assertion is established, with $\mathbf{g}=\mathbf{g}_{1}$ or $\mathbf{g}_{2}$.

Suppose that $\mathbf{g}_{1}(v)=v$ and $\mathbf{g}_{2}(u)=u$. Then $\mathbf{g}_{1}(u) \neq \mathbf{g}_{2}(u), \mathbf{g}_{2}(v)$ and $\mathbf{g}_{1}(v) \neq \mathbf{g}_{2}(u), \mathbf{g}_{2}(v)$. Therefore $\mathbf{g}_{2}^{-1} \mathbf{g}_{1}(u) \neq u, v$ and $\mathbf{g}_{2}^{-1} \mathbf{g}_{1}(v) \neq u, v$. We may take $\mathbf{g}=\mathbf{g}_{2}{ }^{-1} \mathbf{g}_{1}$.

Suppose that $\mathbf{g}_{1}(v)=u=\mathbf{g}_{2}(u)$. Then $\mathbf{g}_{1}{ }^{2}(v)=\mathbf{g}_{1}(u)$, and $\mathbf{g}_{1}{ }^{2}(u) \neq u$. If $\mathbf{g}_{1}^{2}(u) \neq v$, we may take $\mathbf{g}=\mathbf{g}_{1}{ }^{2}$. If $\mathbf{g}_{1}{ }^{2}(u)=v$, then $\mathbf{g}_{1}{ }^{2}(u) \neq \mathbf{g}_{2}(u), \mathbf{g}_{2}(v)$ and $\mathbf{g}_{1}{ }^{2}(v) \neq \mathbf{g}_{2}(u), \mathbf{g}_{2}(v)$. We may take $\mathbf{g}=\mathbf{g}_{2}{ }^{-1} \mathbf{g}_{1}{ }^{2}$. The case that $\mathbf{g}_{1}(v)=v=\mathbf{g}_{2}(u)$ is treated in a similar manner.

Suppose that $\mathbf{g}_{1}(v)=u$ and $\mathbf{g}_{2}(u)=v$. Then $\mathbf{g}_{1}(u) \neq \mathbf{g}_{2}(u), \mathbf{g}_{2}(v)$ and $\mathbf{g}_{1}(v) \neq \mathbf{g}_{2}(u), \mathbf{g}_{2}(v)$. We may take $\mathbf{g}=\mathbf{g}_{2}{ }^{-1} \mathbf{g}_{1}$.

We now know that there is $\mathbf{g} \in \mathbb{G S}$ so that $\mathbf{g}(u) \neq u, v$ and $\mathbf{g}(v) \neq u, v$. Let $z \in L_{\leftrightarrow} \cap U$ and let $\left\{\mathbf{g}_{n}\right\}$ be a sequence in (S) so that $\lim _{n \rightarrow \infty} \mathbf{g}_{n}(p)=z$, for any $p \in D$. The proof of Proposition 11 shows that if $x_{0} \in E$ and $\left\{\mathbf{g}_{n_{k}}\right\}$ is a subsequence of $\left\{\boldsymbol{g}_{n}\right\}$ such that $\left\{\boldsymbol{g}_{n_{k}}\left(x_{0}\right)\right\}$ converges to a point different from $z$, then $\lim _{k \rightarrow \infty} \mathbf{g}_{n_{k}}(x)=z$ for all $x \in E, x \neq x_{0}$. Therefore, there is a subsequence $\left\{\mathbf{g}_{n_{k}}\right\}$ of $\left\{\mathbf{g}_{n}\right\}$ such that either $\lim _{k \rightarrow \infty} \mathbf{g}_{n_{k}}(u)=z$ and $\lim _{k \rightarrow \infty} \mathbf{g}_{n_{k}}(v)=z$, or $\lim _{k \rightarrow \infty} \mathbf{g}_{n_{k}} \mathbf{g}(u)=z$ and $\lim _{k \rightarrow \infty} \mathbf{g}_{n_{k}} \mathbf{g}(v)=z$. Thus, for large $k$, either $\mathbf{g}_{n_{k}} \mathbf{f g}{ }_{n_{k}}{ }^{-1}$ or $\left(\mathbf{g}_{n_{k}} \mathbf{g}\right) \mathbf{f}\left(\mathbf{g}_{n_{k}} \mathbf{g}\right)^{-1}$ has its fixed points in $U$.

We shall say that the fixed points of the loxodromic elements of \&s are dense in $L_{\mathfrak{G}} \times L_{\mathfrak{G}}$, if for any points $z_{1}, z_{2} \in L_{\mathfrak{G}}$ and $E$-neighborhoods $U_{1}, U_{2}$ of these points, there is a loxodromic element in ${ }_{5}$ which has one fixed point in $U_{1}$ and the other in $U_{2}$.

Proposition 12. If (S) contains loxodromic elements, and the elements of (S) do not have a common fixed point in $E$, then the fixed points of the

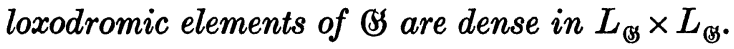

Proof. If the axis of a loxodromic transformation is invariant under (S), then $L_{\mathscr{B}}$ consists of the fixed points of this transformation, and the proposition is established. Otherwise, Lemma 1 can be applied. Suppose $z_{1}, z_{2} \in L_{\mathscr{G}}$ and $U_{1}, U_{2}$ are disjoint $E$-neighborhoods of these points. By Lemma 1 , there is a loxodromic transformation $f_{1} \in \mathbb{G S}$ whose fixed points are in $U_{1}$, and there is an element $g \in \mathbb{S}$, so that $\mathbf{f}_{2}=\mathbf{g f}_{1} \mathbf{g}^{-1}$ has its fixed points in $U_{2}$. Let $\pi$ be an $(n-1)$-dimensional h-plane which is orthogonal to the axis $A_{f_{1}}$, and which intersects $E$ inside $U_{1} . \pi$ separates $D$ into two half-spaces. Let $H_{1}$ be the half-space whose boundary contains $z_{2} . g A_{t_{1}}$ is the axis of $f_{2}=g f_{1} g^{-1}$ and $g(\pi)$ is an h-plane orthogonal to this axis. $\mathbf{g}(\pi)$ is translated along $A_{\mathbf{f}_{2}}$ by the transformations $\mathbf{f}_{\mathbf{2}}{ }^{n}$. Choose $n$ large enough, and with the correct sign, so that $\mathbf{f}_{2}{ }^{n} \mathbf{g}\left(H_{1}\right)=H_{2}$ is contained in $H_{1}$ and $\gamma_{2}=\bar{H}_{2} \cap E$ is contained in $U_{2}$. If $\gamma_{1}=\bar{H}_{1} \cap E$, 
then $\gamma_{1} \supset \gamma_{2}$ and $\mathbf{f}_{2}{ }^{n} \mathbf{g} \gamma_{1}=\gamma_{2}$. By the Brouwer fixed point theorem, there is a fixed point of $\mathbf{f}_{2}{ }^{n} \mathbf{g}$ in $\gamma_{2} \subset U_{2}$. Similarly, if $\gamma_{i}{ }^{\prime}=\overline{E-\gamma_{i}}$, then $\gamma_{2}{ }^{\prime} \supset \gamma_{1}{ }^{\prime}$ and $\left(\mathbf{f}_{2}{ }^{n} \mathbf{g}\right)^{-1} \gamma_{2}{ }^{\prime}=\gamma_{1}{ }^{\prime}$. Thus there is a fixed point of $\mathbf{f}_{2}{ }^{n} \mathbf{g}$ in $\gamma_{1}{ }^{\prime} \subset U_{1}$. Since $\mathrm{f}_{2}{ }^{n} \mathbf{g}$ maps $H_{1}$ onto $H_{2}$, it moves every point on the $\mathrm{h}$-line between the fixed points, and therefore it cannot be elliptic. Thus $\mathbf{f}_{\mathbf{2}}{ }^{n} \mathbf{g}$ is loxodromic, and the proposition is established.

Proposition 13. If $L_{\Theta}=\emptyset$, then the elements of \&S have a common fixed point in $D$.

Proof. $\overline{\Theta S}$ is a closed Lie subgroup of $\mathfrak{Q}$, and $L_{\bar{\circlearrowleft}}=L_{\mathscr{G}}=\emptyset$. For any $z \in D$, the orbit $\overline{\text { SS}} z$ is a closed bounded set (in the hyperbolic metric), so that $\overline{\mathfrak{S}} z$ is compact. Let $\mathfrak{R}=\{\mathbf{g} \in \mathbb{S} \mid \mathbf{g}(z)=z\}$. $\mathfrak{R}$ is a closed subgroup of the orthogonal group, so $\Re$ is compact. The factor space $\bar{\Theta} / \Omega$ is homeomorphic to $\bar{\Xi} z$, so $\bar{\Theta} / \Omega$ is compact. $\bar{\Theta}$ is a fibre space with compact base $(\overline{\mathbb{S}} / \Re)$ and compact fibre $(\Re)$. Therefore $\overline{\mathscr{S}}$ is compact. If $\mu$ is the Haar measure in $\mathbb{S S}, \mu(\overline{\mathbb{S}})<\infty$.

We shall now find it convenient to operate in the Cayley-Klein model of hyperbolic space. This is the set

$$
C=\left\{\left(x_{1}, \ldots, x_{n+1}\right) \mid x_{1}^{2}+\ldots+x_{n}^{2}-x_{n+1}^{2}<0\right\}
$$

in projective space. The transformations in $\mathfrak{L}$ operate as collineations. $C$ is closed under addition in the following sense. If $x=\left(x_{1}, \ldots, x_{n+1}\right)$ and $y=\left(y_{1}, \ldots, y_{n+1}\right)$ are points in $C$, such that $x_{n+1}$ and $y_{n+1}$ are positive, then $x+y \in C$. For

$$
\begin{aligned}
\sum_{i=1}^{n}\left(x_{i}+y_{i}\right)^{2} & =\sum_{i=1}^{n} x_{i}{ }^{2}+\sum_{i=1}^{n} y_{i}{ }^{2}+2 \sum_{i=1}^{n} x_{i} y_{i} \\
& <x_{n+1}{ }^{2}+y_{n+1}{ }^{2}+2\left[\sum_{i=1}^{n} x_{i}{ }^{2}\right]^{\frac{1}{2}}\left[\sum_{i=1}^{n} y_{i}{ }^{2}\right]^{\frac{1}{2}} \\
& <x_{n+1}{ }^{2}+y_{n+1}{ }^{2}+2 x_{n+1} y_{n+1}=\left(x_{n+1}+y_{n+1}\right)^{2} .
\end{aligned}
$$

Let $\mathbf{g}(x)=\left(g_{1}(x), \ldots, g_{n+1}(x)\right)$. If $\mathbf{g} \in \mathfrak{L}$ and $x \in C$, then $\operatorname{sign}\left[g_{n+1}(x)\right]=$ $\operatorname{sign}\left[x_{n+1}\right]$. Let $z \in C$ and $z_{n+1}>0$. Since $\overline{\mathbb{S}} z$ is compact, $g_{k}(z)$ is a bounded, continuous function of $\mathbf{g}$, and the integral $\int_{\bar{G}} \mathbf{g}(z) d \mu$ exists. Define

$$
z_{0}=\int_{\overparen{ङ}} \mathrm{~g}(z) d \mu .
$$

Then $z_{0}$ is a point in the convex closure of $\bar{\Xi} z$, which is fixed under $\overline{\mathbb{S}}$.

Let $\pi$ be an $m$-dimensional h-plane, $1 \leqq m \leqq n-1$. We shall use the following notations. $\mathfrak{L}(\pi)$ is the group generated by the reflections in 
$(n-1)$-dimensional h-planes orthogonal to $\pi$, and $\mathfrak{L}+(\pi)$ is the group generated by the hyperbolic transformations with axes in $\pi$. Thus, $\mathfrak{L}(\pi)$ is the group of rigid motions of $\pi, \Omega^{+}(\pi)$ is the orientation-preserving subgroup, and $\left[\mathfrak{L}(\pi): \mathfrak{L}^{+}(\pi)\right]=2 . \quad \Re(\pi)$ is the group of transformations in $\mathfrak{Q}$ which leave every point of $\pi$ fixed. $\Im(\pi)$ is the group of transformations in $\mathfrak{Q}$ which leave $\pi$ invariant, and $\mathfrak{\Im}^{+}(\pi)$ is the subgroup of transformations which induce orientation-preserving motions in $\pi$, so that $\left[\Im(\pi): \mathfrak{\Im}^{+}(\pi)\right]=2$. It is easy to see that

and

$$
\Im(\pi)=\Re(\pi) \times \mathfrak{L}(\pi),
$$

$$
\mathfrak{S}^{+}(\pi)=\Omega(\pi) \times \mathfrak{L}^{+}(\pi) .
$$

We shall denote by $\theta$ and $\varphi$ the projections of $\Im^{+}(\pi)$ onto $\Re(\pi)$ and $\mathfrak{L}+(\pi)$, respectively. If $\mathscr{S} \subset \mathfrak{\Im}(\pi)$ we shall use the notation $\mathscr{S}^{+}=\mathscr{S} \cap \mathfrak{S}^{+}(\pi)$. The subgroup $\mathbb{B S}^{+}$is of index 2 in $\mathbb{B S}$.

Proposition 14. Let $\mathbb{1 S}$ be a Lie subgroup of $\mathfrak{L}$, such that $L_{\mathfrak{G}}$ contains exactly two points, and let $\lambda=\pi\left[L_{\mathfrak{G}}\right]$ be the $\mathrm{h}$-line between these points. Then one of the following alternatives is true.

1) The component of the identity in $\mathbb{S}$ is contained in $\mathfrak{I}(\lambda)$.

2) $\mathbb{S S}^{+}=\mathfrak{\Re} \times \mathfrak{M}$, where $\mathfrak{\Re}$ is a Lie subgroup of $\mathfrak{R}(\lambda)$, and $\mathfrak{M}$ is a 1-parameter loxodromic group with axis $\lambda$.

Proof. Let $\mathscr{S}_{0}$ be the connected component of $\mathscr{S S}^{+}$(and $\left.\mathbb{S}\right)$, and suppose that $\mathscr{S}_{0}$ is not contained in $\Re(\lambda)$. Let $\mathscr{G}, \mathscr{K}(\lambda), \mathscr{L}(\lambda)$ be the Lie algebras of $\mathscr{S}, \mathfrak{R}(\lambda), \mathfrak{L}(\lambda)$, respectively. There is a negative-definite form $\Phi$ in $\mathscr{K}(\lambda)$, such that ad $K$ is skew-symmetric with respect to $\Phi$, for any $K \in \mathscr{K}(\lambda)$. If dimension $\{\mathscr{K}(\lambda)\}=1$, we can take $\Phi$ to be any negativedefinite form. If dimension $\{\mathscr{K}(\lambda)\}>1$, take $\Phi$ to be the Killing form. $\Phi$ can be extended to $\mathscr{K}(\lambda) \oplus \mathscr{L}(\lambda)$ by letting $\Phi(K, H)=0, \Phi(H, H)=-1$, where $K \in \mathscr{K}(\lambda)$ and $H$ is a chosen element of the 1-dimensional algebra $\mathscr{L}(\lambda)$. The restriction of $\Phi$ to $\mathscr{G}$ is a negative-definite form (which we again denote by $\Phi$ ) such that $\operatorname{ad} G$ is skew-symmetric with respect to $\Phi$, for any $G \in \mathscr{G}$. This implies that every ideal in $\mathscr{G}$ has a complementary ideal. The projection $\varphi: \mathbb{S}^{+} \rightarrow \mathfrak{L}^{+}(\lambda)$ induces a homomorphism $\varphi^{\prime}: \mathscr{G} \rightarrow \mathscr{L}(\lambda)$. Let $\mathscr{K}=\operatorname{kernel}\left(\varphi^{\prime}\right)$, and let $\mathscr{M}$ be the complement of $\mathscr{K}$ in $\mathscr{G}$ with respect to $\Phi$. The assumption that $\mathscr{S}_{0}$ is not contained in $\Re(\lambda)$ implies that $\mathscr{K}$ is a proper ideal in $\mathscr{G}$ and $\mathscr{M}$ is a non-trivial ideal. $\mathscr{G}=$ $\mathscr{K} \oplus \mathscr{M}, \mathscr{K} \subset \mathscr{K}(\lambda), \mathscr{M} \cap \mathscr{K}(\lambda)=\{0\}$ and $\mathscr{M}$ is 1-dimensional.

Let $\mathfrak{\Re}_{0}$ and $\mathfrak{M}$ be the Lie subgroups of $\mathscr{S}_{0}$ which correspond to $\mathscr{K}$ and $\mathscr{M}$, respectively. The elements of $\mathfrak{\Omega}_{0}$ commute with those of $\mathfrak{M}$, and $\mathfrak{S}_{0}=\mathbb{R}_{0} \mathfrak{M}$. If $\mathfrak{M}$ contains no loxodromic elements, then $\mathfrak{S}_{0} \subset \mathfrak{I}(\lambda)$. Therefore $\mathfrak{M}$ is a 1-parameter, loxodromic group. 
Let $\mathfrak{R}=\operatorname{kernel}(\varphi)=\mathbb{S}^{+} \cap \mathfrak{R}(\lambda)$. It is easy to see that $\mathfrak{S}^{+}=\mathfrak{R M}_{\mathfrak{R}}$ and $\mathfrak{R} \cap \mathfrak{M}=\{1\}$. If we show that $\mathfrak{M}$ is a normal subgroup of $\mathbb{S}^{+}$, then it follows that $\mathscr{S}^{+}=\mathfrak{\Omega} \times \mathfrak{M}$. If $\mathbf{g} \in \mathscr{S}^{+}$, the mapping $\mathbf{f} \rightarrow \mathbf{g f g}^{-1}$ induces an automorphism $\mathbf{A}(\mathbf{g})$ of $\mathscr{K}(\lambda) \oplus \mathscr{L}(\lambda)$, which maps $\mathscr{K}(\lambda)$ onto itself. $\mathbf{A}(\mathbf{g})$ preserves the form $\Phi$ in $\mathscr{K}(\lambda)$ and maps $H \in \mathscr{L}(\lambda)$ into itself. Therefore $\mathbf{A}(\mathbf{g})$ preserves the form $\Phi$ in $\mathscr{K}(\lambda) \oplus \mathscr{L}(\lambda)$ and in $\mathscr{G}$. Since $\mathbf{A}(\mathbf{g})$ maps $\mathscr{K}$ onto itself, it maps $\mathscr{M}$ onto itself. Thus the conjugation $\mathbf{f} \rightarrow \mathbf{g f g}^{-1}$ preserves $\mathfrak{M}$, and $\mathfrak{M}$ is normal.

By the centralizer $\mathfrak{E}(\mathbb{S}, \mathfrak{H})$, we shall mean the group

$$
\mathfrak{C}(\mathbb{S}, \mathfrak{H})=\{\mathbf{h} \mid \mathbf{h} \in \mathfrak{H}, \mathbf{g h}=\mathbf{h g} \text { for all } \mathbf{g} \in \mathbb{S}\} \text {. }
$$

We recall that if $S$ is any subset of $E$, then $\sigma[S]$ is the smallest sphere in $E$ which contains $S$, and $\pi[S]$ is the h-plane such that $\sigma[S]=\overline{\pi[S]} \cap E$.

Proposition 15. Suppose that $\mathbb{E}$ is a subgroup of $\mathfrak{L}(\pi)$, where $\pi=\pi\left[L_{\leftrightarrow}\right]$. If $L_{\mathscr{S}}$ contains more than two points, then the centralizer $\mathfrak{C}(\mathfrak{S S}, \mathfrak{L}(\pi))$ is trivial. If, in addition, the elements of $\mathbb{S}$ do not have a common fixed point in $\sigma\left[L_{\mathbb{G}}\right]$, then SS is semi-simple.

Proof. Suppose that $\mathrm{c}$ is an element in the centralizer and $z_{0} \in L_{\mathfrak{B}}$. There is a sequence $\left\{\mathbf{g}_{n}\right\} \subset \mathbb{S}$, so that $\lim _{n \rightarrow \infty} \mathbf{g}_{n}(z)=z_{0}$, for any $z \in D$. Then

$$
z_{0}=\lim _{n \rightarrow \infty} \mathbf{g}_{n}(z)=\lim _{n \rightarrow \infty} \operatorname{cg}_{n} \mathbf{c}^{-1}(z)=\lim _{n \rightarrow \infty} \operatorname{cg}_{n}(z)=\mathbf{c}\left(z_{0}\right) \text {. }
$$

Thus every point in $L_{\mathscr{S}}$ is a fixed point of c. By Proposition 2, every point in $\pi\left[L_{\mathfrak{G}}\right]$ is fixed by $\mathbf{c}$. Since $\mathbf{c} \in \mathfrak{L}(\pi)$, this implies that $\mathbf{c}=1$.

Now suppose that $\mathfrak{N}$ is a commutative, normal subgroup of $\mathbb{S}$. If $L_{\mathfrak{R}}=\emptyset$, then $\mathfrak{R}$ has a common fixed point $z \in D$. Suppose that $z_{0} \in L_{\mathfrak{G}},\left\{\boldsymbol{g}_{k}\right\}$ is a sequence in $\mathbb{S}$, so that $\lim _{k \rightarrow \infty} \mathbf{g}_{k}(z)=z_{0}$, and $\mathbf{n} \in \mathfrak{N}$. The point $\mathbf{g}_{k}(z)$ is fixed under $\mathbf{g}_{k} \mathbf{n} \mathbf{g}_{k}{ }^{-1}$. Since the mapping $\mathbf{n} \rightarrow \mathbf{g}_{k} \mathbf{n} \mathbf{g}_{k}{ }^{-1}$ maps $\mathfrak{N}$ onto itself, $\mathbf{g}_{k}(z)$ is a common fixed point of $\mathfrak{R}$. It follows that $z_{0}$ is a common fixed point of $\mathfrak{N}$. Thus the elements of $\mathfrak{N}$ leave every point of $L_{\text {ळ }}$ and $\pi\left[L_{\mathfrak{G}}\right]$ fixed. Since $\mathfrak{R} \subset \mathfrak{L}(\pi)$, if follows that $\mathfrak{N}=\{1\}$.

If $L_{\mathfrak{R}} \neq \emptyset$, we will show that $L_{\mathfrak{R}}=L_{\mathfrak{G}}$. Suppose that $z_{0} \in L_{\mathfrak{\Re}}$, $\left\{\mathbf{n}_{k}\right\} \subset \mathfrak{R}, \lim _{k \rightarrow \infty} \mathbf{n}_{k}(z)=z_{0}$, and $\mathbf{g} \in \mathbb{E}$. Then

$$
\lim _{k \rightarrow \infty} \mathbf{g n}_{k} \mathbf{g}^{-1}(z)=\lim _{k \rightarrow \infty} \mathbf{g n}_{k}(z)=\mathbf{g}\left(z_{0}\right) \text {. }
$$

Thus $L_{\mathfrak{R}}$ is invariant under $\mathbb{S}$. If $L_{\mathfrak{R}}$ consists of exactly one point, then this point is invariant under $\&$, contradicting the hypothesis. Therefore $L_{\mathfrak{R}}$ is a closed subset of $E$, which contains more than one point, and $L_{\mathfrak{R}}$ is invariant under $\mathfrak{S}$. Proposition 11 implies that $L_{\mathfrak{R}} \supset L_{\mathfrak{G}}$. Since $\mathfrak{R} \subset \mathbb{E}$, 
$L_{\mathfrak{R}} \subset L_{\mathfrak{G}}$, and so $L_{\mathfrak{R}}=L_{\mathfrak{G}}$. Therefore $\pi=\pi\left[L_{\mathfrak{R}}\right]$ and $\mathfrak{R} \subset \mathfrak{L}(\pi)$. As we have already shown, this implies that the centralizer $\mathfrak{E}(\mathfrak{R}, \mathfrak{L}(\pi))$ is trivial. Since $\mathfrak{N}$ is commutative, it is a subgroup of this centralizer, and $\mathfrak{R}=\{\mathbf{1}\}$.

Proposition 16. Let \&S be a Lie subgroup of $\mathfrak{L}+(\pi)$, where $\pi=\pi\left[L_{\mathfrak{G}}\right]$. Suppose that $L_{\mathfrak{G}}$ contains more than two points and the elements of (S) do not have a common fixed point in $\sigma\left[L_{\mathfrak{G}}\right]$. Then either $\mathbb{B S}$ is discrete or $\mathbb{S}=\mathfrak{L}+(\pi)$.

Proof. Suppose that $\left(\mathbb{S}\right.$ is not discrete, and let $\mathscr{S}_{0}$ be the component of the identity in $\mathbb{S}$. $\mathscr{G S}_{0}$ is a non-trivial normal subgroup of $\mathfrak{G S}$. As in the proof of Proposition 15, $L_{\mathfrak{G}_{0}}=L_{\mathfrak{G}}$. Furthermore, the elements of $\mathbb{S}_{0}$ do not have a common fixed point in $\sigma\left[L_{\mathfrak{G}}\right]$. For if $z$ is such a point and $\mathbf{g} \in \mathbb{S S}$, then $\mathbf{g}(z)$ is also a common fixed point of $\mathbb{S S}_{0}$. But the orbit $\mathbb{B S} z$ is dense in $L_{\mathfrak{S}}$, so every point of $L_{\mathfrak{G}}$ and $\pi\left[L_{\mathfrak{G}}\right]$ is fixed under $\mathscr{S}_{0}$, and $\mathscr{G}_{0}=\{1\}$. Proposition 15 implies that $\mathscr{J}_{0}$ is semi-simple with trivial centralizer. Proposition 9 implies that $\mathbb{S}_{0}$ contains the hyperbolic, elliptic and strictly parabolic components of its elements. If we show that $\mathbb{S}_{0}$ contains loxodromic elements, then the above facts and Proposition 12 imply that the fixed points of the hyperbolic elements are dense in $L_{\mathfrak{G}} \times L_{\mathfrak{G}}$.

Suppose that $\mathscr{G}_{0}$ contains no loxodromic elements, and let $\mathscr{G}$ be the Lie algebra of $\mathscr{G}_{0}$. Proposition 5 implies that the Killing form of $\mathscr{L}$ is negative semi-definite in $\mathscr{G}$. The equation $\langle X, X\rangle=0$ holds only if $X$ is strictly parabolic or loxodromic. If $\mathscr{S}_{0}$ contains strictly parabolic elements, then it contains two strictly-parabolic elements $s_{1}$ and $s_{2}$ with different fixed points. But $\mathbf{s}_{1} \mathbf{s}_{2} \mathbf{s}_{1}{ }^{-1} \mathbf{s}_{2}{ }^{-1}$ is hyperbolic (since this is true in the 2-dimensional case). Therefore $\mathfrak{B S}_{0}$ contains no parabolic elements, and the Killing form of $\mathscr{L}$ is negative definite in $\mathscr{G}$. The group $\mathfrak{A}_{\mathfrak{G}_{0}}$, which is isomorphic to $\mathfrak{S H}_{0}$, is a subgroup of the orthogonal group with respect to this form in $\mathscr{G}$. Since $\mathscr{G}_{0}$ is semi-simple, this is a closed subgroup of the orthogonal group, and $\mathscr{G}_{0}$ is compact. But this implies that $L_{\mathfrak{B}_{0}}=\varnothing$.

We now know that the fixed points of the hyperbolic elements of $\mathbb{S}_{0}$ are dense in $L_{\mathscr{G}} \times L_{\mathscr{G}}$. Also, according to Proposition 9, the hyperbolic elements are contained in 1-parameter hyperbolic subgroups of $\mathbb{B S}_{0}$.

We assert that $\mathbb{S Y}_{0}$ contains every hyperbolic transformation whose fixed points are in $L_{\mathfrak{G}}$. Let $\mathbf{h}$ be hyperbolic with fixed points $u, v \in L_{\mathfrak{G}}$ and translation length $\lambda$. We shall suppose that $h$ moves points toward $v$. There is a sequence of hyperbolic elements $\mathbf{h}_{n} \in \mathfrak{S G}_{0}$, so that $\mathbf{h}_{n}$ has fixed points $u_{n}, v_{n}$ and translation length $\lambda, \mathbf{h}_{n}$ moves points towards $v_{n}$, and $\lim _{n \rightarrow \infty} u_{n}=u, \lim _{n \rightarrow \infty} v_{n}=v$. This implies that $\lim _{n \rightarrow \infty} \mathbf{h}_{n}=\mathbf{h}$. By Proposition $10, \mathbb{B S}_{0}$ is closed, so $h \in \mathbb{S}_{0}$. 
We now assert that if $z_{1}, z_{2}$ and $z_{3}$ are any three points in $L_{\leftrightarrow}$, and $\gamma$ is the unique circle containing these points, then $\gamma \subset L_{\mathscr{G}}$. The group $\mathbb{G}_{0}$ contains the 1-parameter subgroup $\mathfrak{S}_{12}$ of hyperbolic transformations with fixed points $z_{1}, z_{2}$. The orbit $\mathfrak{S}_{12} z_{3}$ is the $\operatorname{arc} z_{1} z_{2}$ of $\gamma$ which contains $z_{3}$. If $\mathfrak{S}_{13}$ is the 1-parameter hyperbolic subgroup with fixed points $z_{1}, z_{3}$, then $\mathfrak{S}_{13} \subset \mathfrak{S}_{0}$ and $\mathfrak{S}_{13} z_{2}$ is the arc $z_{1} z_{3}$ of $\gamma$ which contains $z_{2}$. Since $L_{\mathfrak{S}}=L_{\mathfrak{S}_{0}}$ is invariant under $\mathfrak{B S}_{0}$,

$$
\gamma=\left\{z_{1}\right\} \cup \mathfrak{S}_{12} z_{3} \cup \mathfrak{S}_{13} z_{2} \subset L_{\text {GS }} .
$$

Since $L_{\overparen{\Theta}}$ contains every circle through any three of its points, it follows that $L_{\mathfrak{G}}=\sigma\left[L_{\mathfrak{G}}\right]$. Therefore $\mathfrak{G S}_{0}$ contains every hyperbolic transformation in $\mathfrak{L}^{+}(\pi)$. Since the hyperbolic transformations generate $\mathfrak{L}^{+}(\pi)$, it now follows that $\mathbb{S}_{0}=\mathfrak{L}^{+}(\pi)=\mathbb{S}$.

Proposition 17. Let $\pi$ be an $\mathrm{h}$-plane (we assume here that dimension $(\pi) \geqq 2$ ) and let $\mathbb{S}$ be a Lie subgroup of $\mathfrak{\Im}^{+}(\pi)$, such that $\varphi(\mathfrak{S})=\mathfrak{L}^{+}(\pi)$. Then

$$
\text { (S) }=\mathfrak{R} \times \mathfrak{Q}+(\pi),
$$

where $\AA$ is a Lie subgroup of $\Re(\pi)$.

Proof. Let $\mathfrak{R}=\theta(\mathfrak{S})$ and $\mathfrak{H}=\operatorname{kernel}(\theta)$. If $\mathfrak{H}=\{\mathbf{1}\}$, then $\theta$ is an isomorphism, and $\varphi \theta^{-1}$ is a homomorphism of $\mathfrak{R}$ onto $\mathfrak{Q}^{+}(\pi)$. We shall show that such a homomorphism does not exist.

Let $\mathscr{K}, \mathscr{K}(\pi), \mathscr{L}(\pi)$ be the Lie algebras of $\mathfrak{R}, \mathfrak{N}(\pi), \mathfrak{L}^{+}(\pi)$ respectively. The group homomorphism defines an algebra homomorphism $\eta$ of $\mathscr{K}$ onto $\mathscr{L}(\pi)$. Since $\mathscr{\Re}(\pi)$ is an orthogonal group, the Killing form $\Phi$ in $\mathscr{K}(\pi)$ is negative definite. Let $\mathscr{A}=\operatorname{Kernel}(\eta)$ and let $\mathscr{B}$ be the orthogonal complement in $\mathscr{K}$ with respect to $\Phi$. Then $\mathscr{A}$ and $\mathscr{B}$ are ideals of $\mathscr{K}$, and $\mathscr{K}=\mathscr{A} \oplus \mathscr{B}$. The automorphism $\eta$ defines an isomorphism of $\mathscr{B}$ onto $\mathscr{L}(\pi)$. Therefore $\mathscr{B}$ is a simple Lie subalgebra of the Lie algebra of the orthogonal group. For such an algebra, any representation (in particular $\eta: \mathscr{B} \rightarrow \mathscr{L}(\pi)$ ) is equivalent to a representation by skew-symmetric matrices. This contradicts the fact that $\mathfrak{Q}+(\pi)$ contains loxodromic and parabolic elements.

We now know that $\mathfrak{\mathfrak { Q }}=\operatorname{kernel}(\theta)=\mathscr{S} \cap \mathfrak{L}^{+}(\pi)$ is non-trivial. $\mathfrak{H}$ is a normal Lie subgroup of $\mathfrak{S S}$. We assert that $\mathfrak{H}$ is also normal in $\mathfrak{L}^{+}(\pi)$. Let $\mathbf{h} \in \mathfrak{G}$ and $\mathbf{f} \in \mathfrak{L}^{+}(\pi)$. There is $\mathbf{k} \in \mathfrak{R}$, so that $\mathbf{k f} \in \mathfrak{B S}$. Then $\mathbf{f h f}^{-\mathbf{1}=}$ $(\mathbf{k f}) \mathbf{h}(\mathbf{k f})^{-1} \in \mathfrak{H}$. But $\mathfrak{L}^{+}(\pi)$ is simple, so $\mathfrak{E}=\mathfrak{L}^{+}(\pi)$. It now follows that (S) $=\mathfrak{R} \times \mathfrak{L}^{+}(\pi)$.

Proposition 18. Let $\mathfrak{B S}$ be a Lie subgroup of $\mathfrak{Q}$ such that $L_{\mathfrak{G}}$ contains more than two points and the elements of $\mathbb{S S}$ do not have a common fixed point in $E$. Let $\pi=\pi\left[L_{\leftrightarrow}\right]$. Then one of the following alternatives is true. 
1) The component of the identity in $\mathbb{S}$ is contained in $\mathfrak{R}(\pi)$.

2) $\mathbb{S}^{+}=\mathfrak{\Omega} \times \mathfrak{L}^{+}(\pi)$, where $\mathfrak{\AA}$ is a Lie subgroup of $\mathfrak{\Omega}(\pi)$.

Proof. Let $\mathbb{B S}_{0}$ be the component of the identity in $\mathbb{S S}^{(\text {and }} \mathscr{S S}^{+}$) and suppose that $\mathfrak{F}_{0}$ is not contained in $\mathfrak{R}(\pi)$. Then $\mathfrak{M}=\varphi\left(\mathbb{S}^{+}\right)$is a nondiscrete Lie subgroup of $\mathfrak{L}^{+}(\pi)$. Furthermore $L_{\mathfrak{M} t}=L_{\mathfrak{S}}$. For if $z_{0} \in L_{\mathfrak{S}}$, there is a sequence $\left\{\mathbf{g}_{n}\right\} \subset \mathbb{S S}^{+}$so that $\lim _{n \rightarrow \infty} \mathbf{g}_{n}(z)=z_{0}$, for any $z \in D$, $\mathbf{g}_{n}=\mathbf{k}_{n} \mathbf{m}_{n}$, where $\mathbf{k}_{n} \in \mathfrak{R}=\theta\left(\mathfrak{S S}^{+}\right), \mathbf{m}_{n} \in \mathfrak{M}$, and

$$
\lim _{n \rightarrow \infty} \mathbf{m}_{n}(z)=\lim _{n \rightarrow \infty} \mathbf{k}_{n} \mathbf{m}_{n}(z)=z_{\mathbf{0}} \text {. }
$$

If $z_{0} \in L_{\mathfrak{M}}$, let $\lim _{n \rightarrow \infty} \mathbf{m}_{n}(z)=z_{0}$ and $\mathbf{k}_{n} \mathbf{m}_{n} \in \mathbb{S}^{+}$. Then

$$
\lim _{n \rightarrow \infty} \mathbf{k}_{n} \mathbf{m}_{n}(z)=\lim _{n \rightarrow \infty} \mathbf{m}_{n}(z)=z_{\mathbf{0}} \text {. }
$$

Therefore $L_{\mathfrak{M}}=L_{\mathfrak{G}}$, and so $\sigma\left[L_{\mathfrak{M}}\right]=\sigma\left[L_{\mathfrak{S}}\right]=\sigma$. The elements of $\mathfrak{M}$ do not have a common fixed point in $\sigma$, for such a point would be a common fixed point for $\mathbb{S S}$. Proposition 16 implies that $\mathfrak{M}=\mathfrak{R}+(\pi)$. Proposition 17 implies that $\mathbb{B S}^{+}=\mathfrak{\Re} \times \mathfrak{Q}^{+}(\pi)$.

The previous propositions imply the following.

Theorem 1. Let (S) be a connected Lie subgroup of $\mathfrak{2}$. Then one of the following is true.

1) The elements of \&5 have a common fixed point in $D$, and is is conjugate to a Lie subgroup of the orthogonal group.

2). The elements of (S) have a common fixed point in $E$, and IS is conjugate (in $\mathfrak{R}^{\prime}$ ) to a subgroup of the isogonal group of Euclidean space.

3) There is an h-line $\lambda$, so that

$$
\mathfrak{S}=\mathfrak{R} \times \mathfrak{M},
$$

where $\mathfrak{\Re} \mathfrak{\Re}(\lambda)$ and $\mathfrak{M}$ is a 1-parameter, loxodromic group with axis $\lambda$.

4) There is an h-plane $\pi$, so that

where

$$
\mathbb{S}=\mathfrak{R} \times \mathfrak{Q}+(\pi),
$$

5) $\mathbb{B}=\mathfrak{Q}^{+}$.

$$
\mathfrak{R} \subset \Re(\pi)
$$

\section{Discrete subgroups of $\mathfrak{L}$.}

Theorem 1 immediately implies the following.

Theorem 2. If there is no point in $\bar{D}$ or proper sub-sphere in $E$ which is invariant under $\mathbb{S}$, and if there is a non-empty open subset $U$ of $\mathfrak{L}^{+}$such that $U \cap \mathbb{S}=\emptyset$, then $\mathbb{B S}$ is discrete. 
If dimension $\{D\}$ is even, $\mathbb{E}$ is the set of elliptic elements of $\mathfrak{L}$, and $U$ is any neighborhood of the identity, then $U \cap \mathbb{E}$ contains an open set. This is not true for odd dimension, because orthogonal transformations near the identity have an axis. Therefore an elliptic transformation near the identity can be approximated by loxodromic transformations.

Corollary 1. Suppose that dimension $\{D\}$ is even. If there is no point or proper sub-sphere in $E$ which is invariant under (S), and if the elliptic transformations of $\mathbb{S}$ do not have the identity as a limit point, then \&S is discrete.

Corollary 2. If dimension $\left\{\pi\left[L_{\mathfrak{K}}\right]\right\}$ is even, the elements of (I) do not have a common fixed point in $\sigma\left[L_{\mathbb{G}}\right]$, and the elliptic transformations of $B 5$ do not have the identity as a limit point, then (5) is discrete.

Proof. (S) has no invariant sub-sphere of $\sigma\left[L_{\mathfrak{G}}\right]$. If $\sigma$ is such a sphere, then $\sigma$ is a closed invariant set containing more than one point. Therefore $\sigma \supset L_{\mathscr{G}}$ and $\sigma \supset \sigma\left[L_{\mathfrak{G}}\right]$. The group $\varphi\left(\mathscr{S}^{+}\right)$is a subgroup of $\mathfrak{L}^{+}(\pi)$ which satisfies the hypotheses of Corollary 1 (replacing $D, E$ by $\pi\left[L_{\mathfrak{G}}\right], \sigma\left[L_{\mathfrak{G}}\right]$ ). Therefore $\varphi\left(\mathscr{S}^{+}\right)$is discrete. Suppose there is a sequence $\left\{\boldsymbol{g}_{n}\right\} \subset \mathbb{S}^{+}$such that $\lim _{n \rightarrow \infty} \mathbf{g}_{n}=\mathbf{1}$. Then $\mathbf{g}_{n}=\mathbf{k}_{n} \mathbf{f}_{n}$, where $\mathbf{k}_{n} \in \theta\left(\mathbb{S}^{+}\right), \mathbf{f}_{n} \in \varphi\left(\mathscr{S}^{+}\right)$and $\lim _{n \rightarrow \infty} \mathbf{k}_{n}=1=\lim _{n \rightarrow \infty} \mathbf{f}_{n}$. Since $\varphi\left(\mathscr{S H}^{+}\right)$is discrete, it follows that for large $n, \mathbf{f}_{n}=1$ and $\mathbf{k}_{n} \in \mathbb{B S}^{+}$. Since the elliptic elements of $\mathbb{E S}$ do not converge to the identity, $\mathbf{k}_{n}=1$ and $\mathbf{g}_{n}=1$ for large $n$.

There are some open sets in $\mathfrak{L}$ which have a non-trivial intersection with almost all Lie subgroups. We shall give two examples of this. If $\mathbf{f} \in \mathfrak{L}$, let $T(\mathbf{f})=\operatorname{trace}(\mathbf{f})$ and $N(\mathbf{f})=\lambda^{2}-\Sigma \theta_{k}^{2}$, where $\lambda$ is the translation length $\left(\lambda=0\right.$, if $\mathbf{f}$ is elliptic or parabolic), and $\theta_{k}$ are the angles of $f$. Then the sets $U_{T}=\{\mathbf{f} \mid T(\mathbf{f})<n+1\}$ and $U_{N}=\{\mathbf{f} \mid N(\mathbf{f})<0\}$ are open sets which have a non-trivial intersection with every elliptic group and every group $\mathfrak{L}(\pi)$, where dimension $(\pi) \geqq 2$. This implies the following.

Theorem 3. If the elements of $\mathbb{S S}$ do not leave a point or pair of points in $E$ invariant, and if there is a neighborhood $U$ of the identity, such that $U \cap U_{T} \cap \mathbb{S}=\varnothing$ or $U \cap U_{N} \cap \mathbb{S}=\varnothing$, then $\mathbb{S}$ is discrete.

Note that in the 2-dimensional case, either of the conditions $T(\mathbf{f})<n+1$ or $N(\mathbf{f})<0$ characterizes the elliptic transformations. Thus, Theorem 3 is a close analogue of Theorem $B$. The result concerning the condition $U \cap U_{N} \cap \mathbb{S}=\varnothing$ (with the additional condition that \&S contains no parabolic elements) also follows from Theorem $\mathrm{C}$.

Corollary 2 implies the following analogue of Nielsen's theorem:

If $\&$ is a loxodromic group such that dimension $\left\{\pi\left[L_{\text {G }}\right]\right\}$ is even, then (S) is discrete. 
(Note that if $f$ and $g$ are loxodromic transformations with exactly one fixed point, then $f g f^{-1} g^{-1}$ is parabolic. Therefore a loxodromic group with more than one axis does not have a common fixed point.)

It might be thought that any loxodromic group with more than one axis is discrete. We shall give a counter-example of this in the 3-dimensional case.

It is convenient to take, as hyperbolic space, the upper halfspace $\left\{\left(x_{1}, x_{2}, x_{3}\right) \mid x_{3}>0\right\}$. Each orientation-preserving rigid motion is the extension of a unique linear fractional transformation.

$$
\mathbf{f}(z)=\frac{a z+b}{c z+d}, \quad a d-b c=1,
$$

in the plane $x_{3}=0$. The extension of $\mathbf{f}$ is loxodromic if and only if $a+d$ is complex.

Let $\left\{U_{n} \mid n=1,2, \ldots\right\}$ be a basis for the open sets in Euclidean space $R^{6}$. In $U_{1}$ there is a point $\mathbf{P}_{1}=\left(a_{1}{ }^{\prime}, a_{1}{ }^{\prime}, b_{1}{ }^{\prime}, b_{1}{ }^{\prime \prime}, c_{1}{ }^{\prime}, c_{1}{ }^{\prime \prime}\right)$ whose components are independent transcendentals (over the rational field). In $U_{2}$ there is a point $\mathbf{P}_{2}=\left(a_{2}{ }^{\prime}, a_{2}{ }^{\prime \prime}, b_{2}{ }^{\prime}, b_{2}{ }^{\prime \prime}, c_{2}{ }^{\prime}, c_{2}{ }^{\prime \prime}\right)$, such that the components of $\mathbf{P}_{1}$ and $\mathbf{P}_{\mathbf{2}}$ are independent transcendentals. Continuing in this way, we can obtain an everywhere dense set of points $\mathbf{P}_{n}=\left(a_{n}{ }^{\prime}, a_{n}{ }^{\prime \prime}, b_{n}{ }^{\prime}, b_{n}{ }^{\prime \prime}, c_{n}{ }^{\prime}, c_{n}{ }^{\prime \prime}\right)$, so that the set of all components consists of independent transcendentals. Let

Let

$$
\begin{array}{ll}
a_{n}=a_{n}{ }^{\prime}+i a_{n}{ }^{\prime \prime}, & b_{n}=b_{n}{ }^{\prime}+i b_{n}{ }^{\prime \prime}, \\
c_{n}=c_{n}{ }^{\prime}+i c_{n}{ }^{\prime \prime}, & d_{n}=\frac{1+b_{n} c_{n}}{a_{n}} .
\end{array}
$$

$$
\mathbf{f}_{n}=\left(\begin{array}{ll}
a_{n} & b_{n} \\
c_{n} & d_{n}
\end{array}\right)
$$

and let $\mathfrak{F}$ be the group generated by the $\mathfrak{f}_{n}$. Obviously $\mathfrak{F}$ is everywhere dense in the unimodular group. We assert that every element of $\mathfrak{F}$ (besides the identity) is loxodromic. That is to say, we will show that if

$$
\mathbf{f}=\left(\begin{array}{ll}
a & b \\
c & d
\end{array}\right)
$$

is an element of $\mathfrak{F}$, then either $T(\mathbf{f})=a+d$ is complex, or $\mathbf{f}=\mathbf{1}$. $\mathbf{f}$ is a word $w\left(\mathbf{f}_{k_{1}}, \mathbf{f}_{k_{2}}, \ldots, \mathbf{f}_{k_{n}}\right)$ and $T(\mathbf{f})$ is a rational function

$$
R\left(a_{k_{1}}, b_{k_{1}}, c_{k_{1}}, \ldots, a_{k_{n}}, b_{k_{n}}, c_{k_{n}}\right) \text {. }
$$

Let $R=U+i V$, where $U$ and $V$ are real, rational functions. If $V=0$ for $\mathbf{f}$, then because of the transcendental independence, $V$ is identically zero. Then, since $R$ is analytic, $R=r$ (a real constant). This means 
that if $\mathbf{g}_{1}, \mathbf{g}_{2}, \ldots, \mathbf{g}_{n}$ are any unimodular matrices, and $\mathbf{g}=w\left(\mathbf{g}_{1}, \mathbf{g}_{2}, \ldots, \mathbf{g}_{n}\right)$, then $T(\mathbf{g})=r$.

If we take $\mathrm{g}_{1}=\mathrm{g}_{2}=\ldots=\mathrm{g}_{n}=1$, then $\mathrm{g}=1$ and $T(\mathrm{~g})=2$. Therefore $r=2$. If $\mathbf{g}_{1}, \ldots, \mathbf{g}_{n}$ are free generators of a free, hyperbolic group, then $T(\mathrm{~g})=2$ implies that

$$
\mathbf{g}=w\left(\mathbf{g}_{1}, \mathbf{g}_{2}, \ldots, \mathbf{g}_{n}\right)=\mathbf{1},
$$

and the word $w$ can be freely reduced to the identity. Thus

$$
\mathbf{f}=w\left(\mathbf{f}_{k_{1}}, \mathbf{f}_{k_{2}}, \ldots, \mathbf{f}_{k_{n}}\right)=1 .
$$

This also shows that $\mathfrak{F}$ is a free group.

\section{REFERENCES}

1. W. T. van Est, $A$ generalization of a theorem of J. Nielsen concerning hyperbolic groups, Doctoral dissertation, University of Utrecht, 1950.

2. W. Fenchel and J. Nielsen, On discontinuous groups of isometric transformations of the non-Euclidean plane, Studies and Essays presented to R. Cousant on his 60th birthday January 8, 1948, New York, 1948, 117-128.

3. J. Nielsen, Uber Gruppen linearer Transformationen, Mitt. Math. Ges. Hamburg 8 (1940), 82-104.

4. C. L. Siegel, Bemerkung zu einem Satze von Jakob Nielsen, Mat. Tidsskr. B 1950, 66-70.

5. Séminaire "Sophus Lie», le anné (École Normale Supérieure): 1954-55, Théorie des algèbres de Lie. Topologie des groupes de Lie, Paris, 1955, No. 6, M. Lazard, Théorie des répliques. Critère de Cartan.

6. E. B. Van Vleck, On the combination of non-loxodromic substitutions, Trans. Amer. Math. Soc. 20 (1919), 299-312.

BROWN UNIVERSITY, PROVIDENCE, R.I., U.S.A. 